\title{
Epoxide-Assisted Alumina Aerogels by Rapid Supercritical Extraction
}

\author{
Stephen J. Juhl, Nicholas J.H. Dunn, Mary K. Carroll*
}

Department of Chemistry

Ann M. Anderson, Bradford A. Bruno, Jose E. Madero, Michael S. Bono Jr.

Department of Mechanical Engineering

Union College

Schenectady NY 12308

USA

*= corresponding author: carrollm@union.edu

\begin{abstract}
Aerogels offer a potential alternative to noble metals that could reduce both the cost and environmental impact associated with catalytic converter production. The environmental impact of the production of aerogel catalysts could be further reduced by using a rapid supercritical extraction (RSCE) technique, which reduces the time and solvent waste associated with aerogel preparation. Alumina aerogels, which have shown activity in catalyzing exhaust processing reactions, were prepared using an epoxide-assisted gelation technique with RSCE processing in a contained mold in a hydraulic hot press. Samples were characterized by FTIR, XRD, SEM, EDX, nitrogen adsorption porosimetry and pycnometry. Solvent characterization by GC-MS headspace analysis shows that excess propylene oxide and chloropropanol products of an irreversible epoxide ring-opening reaction are present in the alumina gel following gelation, but can be removed via solvent exchange. Alumina aerogels with surface areas as high as $790 \mathrm{~m}^{2} / \mathrm{g}$ and bulk densities as low as $0.05 \mathrm{~g} / \mathrm{mL}$ were prepared. Preliminary characterization of these aerogels, utilizing a catalytic test bed and a simulated emissions gas blend, demonstrates that they have moderate ability for removal of hydrocarbons, carbon monoxide and nitrogen oxide.
\end{abstract}

\section{KEYWORDS}

Alumina aerogels; catalytic aerogels; rapid supercritical extraction 


\section{INTRODUCTION}

The goal of this work was to prepare and characterize alumina-based aerogel materials for eventual application in automobile exhaust-cleaning reactions. Current automotive catalytic converter technology relies on noble metals such as platinum and palladium to perform such reactions, including $\mathrm{NO}_{\mathrm{x}}$ reduction, $\mathrm{CO}$ conversion and the combustion of unburnt hydrocarbons. A washcoat containing platinum, palladium and/or rhodium is supported on a ceramic honeycomb to maximize the available reactive surface area in the converter. The use of noble metals is the main drawback to the current catalytic converter design; the mining and processing of these metals is damaging to the environment, which partially offsets the environmental benefits of using a converter. ${ }^{1}$

Aerogels have favorable properties for catalyzing gas-phase reactions. ${ }^{2}$ They have high specific surface area (e.g., 600-800 $\mathrm{m}^{2} / \mathrm{g}$ for alumina aerogels ${ }^{3,4}$ ), along with high thermal stability and tunable chemical composition, all of which make them appealing for applications in heterogeneous catalysis. ${ }^{2}$ Aerogels have been used as catalysts or catalytic supports in a variety of gas-phase catalytic applications as summarized in Table 1. The high surface area increases the number of exposed active sites for reaction, thereby increasing the efficiency of the reaction with respect to the gas flow.

Silica and alumina aerogels are most often used as supports for catalytically active species, although both materials have also demonstrated catalytic activity on their own. Alumina aerogels have been used in alcohol dehydration to form ethers ${ }^{5}$ and they have been used as NOx reduction catalysts with some success. ${ }^{6}$ Silica aerogels reduced under a hydrogen stream have been used for cracking cumene to generate benzene and propane. $^{7}$

Copper and vanadium supported on silica aerogels have demonstrated reductive activity for the conversion of $\mathrm{NO}$ to $\mathrm{N}_{2}$ and $\mathrm{H}_{2} \mathrm{O} .{ }^{8}$ Copper-silica aerogels have also been used for the reduction of NO in the presence of hydrocarbons, ${ }^{8}$ a reaction of interest to the automotive industry because it doesn't use $\mathrm{NH}_{3}$ as the reductant. Mixed silica and alumina aerogels have been used for the oligomerization of propene to polypropylene $^{9}$, and mixed titania-silica aerogels supporting rhodium have been used in the hydrogenation of benzene ${ }^{10}$. Nickel-alumina aerogels have proven particularly versatile as catalysts, and 
have shown activity for $\mathrm{CO}$ and $\mathrm{NO}$ conversion, hydrogenation, methanization, isomerization and dehydrogenation. ${ }^{11,12}$ The $\mathrm{CO}$ conversion and $\mathrm{NO}_{\mathrm{x}}$ reduction reactions over alumina are of particular interest because such reactions are performed in automotive catalytic converters. Silica and alumina aerogels have been used as supports for palladium in automotive combustion applications; ${ }^{13,14}$ however, we are not aware of prior results demonstrating the applicability of aerogel materials as three-way catalysts.

Table 1: Some Catalytically Active Aerogels Described in the Literature

\begin{tabular}{|c|c|c|c|c|}
\hline Aerogel & Reaction & Reactant & Product & Ref \\
\hline $\mathrm{Al}_{2} \mathrm{O}_{3}$ & dehydration & $\mathrm{CH}_{3} \mathrm{OH}$ & $\mathrm{CH}_{3} \mathrm{OCH}_{3}$ & Tleimet-Manzalji et al. \\
\hline $\mathrm{Al}_{2} \mathrm{O}_{3}$ & reduction & $\mathrm{NO}, \mathrm{NH}_{3}$ & $\mathrm{~N}_{2}, \mathrm{H}_{2} \mathrm{O}$ & Hirashima et al. ${ }^{6}$ \\
\hline $\mathrm{SiO}_{2}$ & cracking & cumene & benzene, propane & Rouenat $^{7}$ \\
\hline $\mathrm{Cu}-\mathrm{SiO}_{2}$ & reduction & $\mathrm{NO}, \mathrm{C}_{3} \mathrm{H}_{8}, \mathrm{C}_{3} \mathrm{H}_{6}$ & $\mathrm{~N}_{2}, \mathrm{H}_{2} \mathrm{O}$ & Owens $^{8}$ \\
\hline $\mathrm{V}-\mathrm{SiO}_{2}$ & reduction & $\mathrm{NO}, \mathrm{C}_{3} \mathrm{H}_{8}, \mathrm{C}_{3} \mathrm{H}_{6}$ & $\mathrm{~N}_{2}, \mathrm{H}_{2} \mathrm{O}$ & Owens $^{8}$ \\
\hline $\mathrm{SiO}_{2}-\mathrm{Al}_{2} \mathrm{O}_{3}$ & oligomerization & propene & polypropylene & Willey et al. ${ }^{9}$ \\
\hline $\mathrm{Rh}-\mathrm{TiO}_{2}-\mathrm{SiO}_{2}$ & hydrogenation & benzene & cyclohexane & Cauqui et al. ${ }^{10}$ \\
\hline $\mathrm{Ni}-\mathrm{Al}_{2} \mathrm{O}_{3}$ & conversion & $\mathrm{CO}$ and $\mathrm{NO}$ & $\mathrm{CO}_{2}$ and $\mathrm{N}_{2}$ & Krompiec $^{11}$ \\
\hline $\mathrm{Ni}-\mathrm{Al}_{2} \mathrm{O}_{3}$ & isomerization & 1-butene & cis/trans-2-butene & Krompiec $^{11}$ \\
\hline $\mathrm{Ni}-\mathrm{Al}_{2} \mathrm{O}_{3}$ & reformation & $\mathrm{CO}_{2}, \mathrm{CH}_{4}$ & $\mathrm{CO}, \mathrm{H}_{2}$ (syngas) & Hao et al. ${ }^{12}$ \\
\hline $\mathrm{Pd}-\mathrm{Al}_{2} \mathrm{O}_{3}$ & automotive & $\mathrm{CO}, \mathrm{NO}, \mathrm{O}_{2}$ & $\mathrm{CO}_{2}, \mathrm{~N}_{2}$ & Hoang et al. ${ }^{13}$ \\
\hline Pd- $\mathrm{SiO}_{2}-\mathrm{Al}_{2} \mathrm{O}_{3}$ & combustion & $\mathrm{CH}_{4}$ & $\mathrm{CO}_{2}, \mathrm{H}_{2} \mathrm{O}$ & Mizumisha $\&$ Hori $^{14}$ \\
\hline
\end{tabular}

We have developed a rapid supercritical extraction (RSCE) method for making aerogels. ${ }^{15,16}$ The method utilizes a contained metal mold, high temperature gaskets and a hydraulic hot press. The aerogel liquid precursors are poured into the mold which is then sandwiched by the gasket material and placed between the hot press platens. The hot press restraining force seals the mold while heating. Upon reaching the supercritical state, the hot press controllably releases the supercritical fluid and then cools the mold at a controlled rate. When cooled to room temperature, the press opens and an aerogel results. This method has been used to make a variety of aerogels including silica, ${ }^{15,16}$ titania, ${ }^{17}$ titania-silica, ${ }^{17}$ alumina, ${ }^{4}$ nickelalumina ${ }^{18}{ }^{\text {cobalt alumina }}{ }^{19}$ and hydrophobic silica ${ }^{20,21}$ aerogels. Aerogels can be prepared in as little as 3 $\mathrm{hrs}^{22}$. The method is inherently safe (there is very little excess solvent) and can be easily automated.

We previously developed an RSCE method for production of alkoxide-based alumina aerogels. ${ }^{4}$ The recipe required careful, temperature controlled mixing of the precursors: aluminum isopropoxide 
(AIP), methanol, water and nitric acid. While it took $15-50 \mathrm{hr}$ to form the alumina sol-gel network, it required only a 7.5-h RSCE extraction process. By varying the catalyst concentration we were able to produce aerogels with surface areas of $460-840 \mathrm{~m}^{2} / \mathrm{g}$ and densities from 0.025 to $0.079 \mathrm{~g} / \mathrm{cm}^{3}$. Although the resulting aerogels had favorable properties, the alkoxide-based approach has some disadvantages. Metal alkoxide precursors are very reactive ${ }^{3}$, sometimes reacting when subjected to light, heat or moisture. ${ }^{23}$ Because of this, some alkoxide-based recipes need to be fabricated in a glove box. ${ }^{24}$ Alkoxides are not commercially available for most transition metals; those that are available are relatively expensive. Many alkoxide recipes require hours of mixing in order to dissolve the alkoxide, hydrolyze it, and condense the resulting hydroxides.

Aerogel recipes have been developed which utilize metal salts such as chlorides and nitrates instead of more reactive alkoxides. Many of these recipes utilize an epoxide proton scavenging technique as seen in Scheme $1 .^{3}$ When a metal salt is dissolved in water, the positively charged metal ion attracts the oxygen atoms in the water. This attraction is frequently strong enough to ionize the water, creating metal hydroxides which can then polymerize to form a gel as with the alkoxide recipes. The released protons from this reaction make the water more acidic, normally causing the solution to reach equilibrium before gelation can occur. When epoxides such as propylene oxide are added to the solution, they consume the protons released by the hydrolysis reaction and allow the reaction to proceed to condensation and gelation. ${ }^{3}$ The essential reactions involved in producing cross-linked metal oxide complexes are shown in Scheme 1. Once the sol-gel matrix has formed, the solvent can be supercritically extracted to yield an aerogel. 


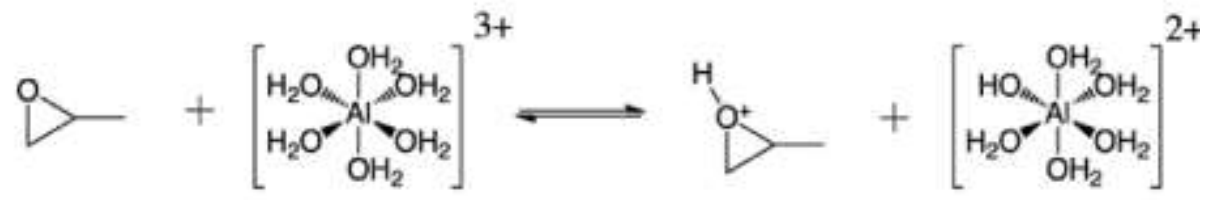

propylene oxide aluminum hexahydrate

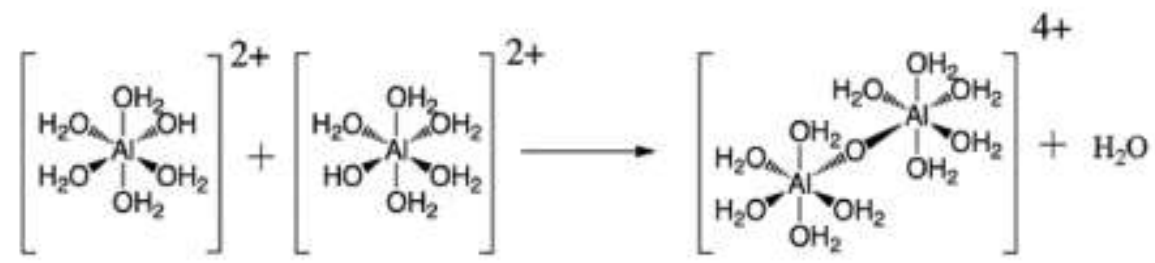

Scheme 1. Proposed mechanism for epoxide-assisted aluminum oxide gel formation, as in Baumann et al. ${ }^{3}$

In this work, we have modified the Baumann et al. epoxide-assisted recipe ${ }^{3}$ to produce alumina aerogels using the RSCE process. We studied the effects of (a) solvent type (ethanol, methanol and 2- propanol), (b) the addition of water to the recipe and (c) solvent exchanges. The following sections describe our experimental approach, characterization methods and results.

\section{EXPERIMENTAL}

\section{Materials}

Aerogels were synthesized by the epoxide-assisted method. ${ }^{3} \mathrm{AlCl}_{3} \cdot 6 \mathrm{H}_{2} \mathrm{O}$ (Fisher, $>99 \%$ purity) was used as the aluminum source for all of the synthesized aerogels. Solvent alcohols employed included methanol (Fisher, $\geq 99.8 \%$ ) ethanol (denatured ethyl alcohol and Pharmco-AAPER, 200 proof, both purchased from Fisher Scientific) and 2-propanol (Fisher, $\geq 99.5 \%$ ). Propylene oxide (Sigma Aldrich, 99\%; safety note: toxic and a probable human carcinogen) was used as the gelation agent for all recipes and deionized water was acquired in-house. 


\section{Gel Preparation}

Table 2 shows the quantities of reagents that were used for each gel recipe. To produce alumina gels, $2.96 \mathrm{~g}$ of $\mathrm{AlCl}_{3} \cdot 6 \mathrm{H}_{2} \mathrm{O}$ was dissolved in $20 \mathrm{~mL}$ of solvent (ethanol, methanol or 2-propanol) in a 50$\mathrm{mL}$ beaker. Then $9.48 \mathrm{~mL}$ of propylene oxide was added to the clear solution, which was stirred with a magnetic stir bar until a rigid gel formed (ca. $3 \mathrm{~min}$ ). The beaker containing the resulting transparent gel was covered with parafilm and the gel was left to set for $24 \mathrm{~h}$. Next, the gel was gently broken into several pieces (1-4 $\mathrm{mL}$ in size) and a solvent exchange was performed. All solvent exchanges were performed by decanting any excess solvent from the beaker and adding $20 \mathrm{~mL}$ of fresh solvent to the gel. The beaker was then covered with parafilm and the gel was allowed to exchange for $24 \mathrm{~h}$. In some cases, additional solvent exchanges were performed. The original solvent used in the formation of the gel was used as the solvent exchange medium except in the case of ethanol; denatured ethyl alcohol was used for gelation, and absolute ethanol was used for the solvent exchanges.

Table 2: Recipes for the Preparation of Gels.

\begin{tabular}{|c|c|c|c|c|c|}
\hline Recipe & Solvent(s) Employed & $\begin{array}{c}\text { Alcohol } \\
(\mathrm{mL})\end{array}$ & $\mathrm{H}_{2} \mathrm{O}(\mathrm{mL})$ & $\begin{array}{c}\mathrm{AlCl}_{3} \cdot 6 \mathrm{H}_{2} \mathrm{O} \\
(\mathrm{g})\end{array}$ & $\begin{array}{c}\text { Propylene Oxide } \\
(\mathrm{mL})\end{array}$ \\
\hline 1 & Methanol & 20 & - & 2.96 & 9.48 \\
\hline 2 & Ethanol & 20 & - & 2.96 & 9.48 \\
\hline 3 & Ethanol/ $\mathrm{H}_{2} \mathrm{O}$ & 10 & 10 & 2.96 & 9.48 \\
\hline 4 & 2-Propanol & 20 & - & 2.96 & 9.48 \\
\hline
\end{tabular}

\section{Rapid Supercritical Extraction}

After the final solvent exchange, the gel was ready to undergo RSCE. ${ }^{15,16}$ The sol-gel fragments were divided evenly between the wells of a stainless steel mold, with the remaining space in each well filled with the solvent alcohol from the preparation recipe. The mold measured $12.6 \times 12.6 \times 1.8 \mathrm{~cm}$, and had four circular wells measuring $4.0 \mathrm{~cm}$ in diameter and $1.5 \mathrm{~cm}$ in depth. The filled mold was placed in a hydraulic hot press and sealed with graphite and stainless steel foil. To seal the contents of the stainless steel mold, the hot press immediately increased the restraining force on the mold to $200 \mathrm{kN}$. Subsequently, the temperature was raised at a rate of $2.2^{\circ} \mathrm{C} / \mathrm{min}$ to a temperature above the supercritical 
point of the alcohol solvent (typically $\sim 250^{\circ} \mathrm{C}$ ). The hot press maintained the mold at the upper temperature and pressure for $30 \mathrm{~min}$ to allow the contents of the mold to come to thermal equilibrium. Next, the restraining force was lowered at a rate of $4.4 \mathrm{kN} / \mathrm{min}$ down to $4.4 \mathrm{kN}$, and the temperature was maintained for 15 min to permit the escape of the supercritical solvent. Finally, the temperature was decreased to $32^{\circ} \mathrm{C}$ at a rate of $2.2^{\circ} \mathrm{C} / \mathrm{min}$ to cool the aerogel and mold prior to removal from the hot press. The total time for the extraction was about $5 \mathrm{hr}$.

\section{Physical Characterization}

Fourier-transform infrared spectroscopy (FTIR) was performed using a Thermo Nicolet Avatar 330 FTIR Spectrophotometer with a Smart Orbit attenuated total reflectance (ATR) attachment. Each sample was crushed prior to analysis. Spectra were taken over the range of $4000-400 \mathrm{~cm}^{-1}$ with 32 averaged scans at $4-\mathrm{cm}^{-1}$ resolution against a background of air.

Powder x-ray diffraction (XRD) patterns were acquired using a Phillips PW-1840 X-ray diffraction instrument employing a $\mathrm{Co}-\mathrm{K} \alpha(\lambda=1.79 \mathrm{~nm})$ radiation source. Each sample was crushed into a powder and packed into an open-backed aluminum sample holder. Diffraction patterns were taken over a $2 \theta$ range of $10^{\circ}$ to $100^{\circ}$ while using a tube voltage of $45 \mathrm{keV}$ and a $35-\mathrm{mA}$ current.

Because the aerogels were too irregular in shape to permit direct volume measurement, bulk density measurements were estimated using aerogel pieces crushed to a coarse powder and placed into a disposable cuvette. The volume and mass of the aerogel powder were measured and bulk density was estimated. This method slightly overestimates the volume (and underestimates the density) of the sample because it does not account for the spaces between aerogel pieces in the cuvette. Skeletal density measurements of finely ground samples were made using a Micromeritics AccuPyc 2020 gas pycnometer, taking repeated measurements until the last four trials agreed with each other within $0.1 \mathrm{~g} / \mathrm{mL}$. The average of these four trials was reported for each sample. The number of measurements taken varied due to different amounts of adsorbed material on the aerogel samples. 
Nitrogen physisorption measurements were taken on a Micromeritics ASAP 2010 gas adsorption system. Aerogel samples were crushed to a coarse powder by a mortar and pestle and then $0.1-0.2 \mathrm{~g}$ of aerogel sample was placed in a glass sample tube. The sample was degassed at $90^{\circ} \mathrm{C}$ for $1 \mathrm{~h}$ and then $200^{\circ} \mathrm{C}$ for $5-12 \mathrm{~h}$ to remove adsorbed species and then massed before testing. BJH pore distribution data was acquired using a 20-s equilibration time to minimize the effects of compression at high pressure. ${ }^{26}$ BET surface area data was acquired using either 5 or $20 \mathrm{sec}$ equilibration using 12 data points taken at partial pressures ranging from $0.01-0.30$. The BJH pore size distribution analysis was calculated using the desorption isotherm.

Scanning electron microscope (SEM) images were taken with a Zeiss EVO50 Scanning Electron Microscope. Pieces of each aerogel sample were placed on carbon tape and then characterized by taking images on $100-\mu \mathrm{m}, 10-\mu \mathrm{m}, 2-\mu \mathrm{m}$, and $1-\mu \mathrm{m}$ scales. Typically a $4.00-\mathrm{keV}$ accelerating voltage and a $20-$ $\mathrm{mA}$ beam current were used in conjunction with a spot size of $250-350$ angstroms $(\AA)$ to obtain highmagnification images. Energy-Dispersive X-ray Spectroscopy (EDX) element mapping was performed using a Bruker Quantax 200 EDX system with a Peltier-cooled XFlash silicon drift detector attachment. Typical parameters for EDX were a $629-\AA$ spot size, an $8.5-\mathrm{mm}$ working distance, and a $16-\mathrm{keV}$ accelerating voltage.

\section{Solvent Characterization}

To investigate the chemical impurities present in the solvent of an alumina gel produced by the epoxide-assisted mechanism, an alumina aerogel was made using eight additional solvent exchanges. During each solvent exchange, the excess solvent was poured into a separate sample vial for testing. Gas chromatography-mass spectrometry (GC-MS) headspace analysis was then used to identify the volatile components present in the solvent after exchange. The GC-MS headspace analysis chromatographs and mass spectra were acquired using an Agilent Technologies 5973 inert Gas Chromatograph/Mass Spectrometer System, consisting of a 6890N Network GC System with a 5973 Series Mass Selective Detector, and employing a 7683 Series Autosampler and a 7863 Series Injector. For testing, a GC-MS 
sample vial was filled with $\sim 1.5-\mathrm{mL}$ of solvent-exchange solution. A 3.0- $\mu \mathrm{L}$ sample of the headspace above the room-temperature solvent-exchange sample was taken and splitless injection was employed. The injection port was held at $250^{\circ} \mathrm{C}$. The Agilent Technologies HP-5MS GC column (30-m length by $0.25-\mathrm{mm}$ diameter by $0.25-\mu \mathrm{m}$ film thickness) was pressurized to $7 \mathrm{psi}$ and utilized a flow rate of 1 $\mathrm{mL} / \mathrm{min}$ of helium gas. During testing, the oven was set to $40^{\circ} \mathrm{C}$ for $8 \mathrm{~min}$, then increased at a rate of $10^{\circ} \mathrm{C} / \mathrm{min}$ to $80^{\circ} \mathrm{C}$ and held at this temperature for $5 \mathrm{~min}$. Finally, the oven was heated to $120^{\circ} \mathrm{C}$ to purge the column.

\section{Calcination Experiments}

We performed calcination experiments on an alumina aerogel by heating the samples to temperatures from 200 to $1100^{\circ} \mathrm{C}$ and performing XRD analysis. First, a diffraction pattern of an unheated sample was taken. Then, the sample was placed in a ceramic crucible and put in an oven for calcination at $200^{\circ} \mathrm{C}$. After two hours, the oven was turned off and the sample was allowed to cool. Once the sample was cool enough to handle, a new diffraction pattern was taken, by the method described above. This procedure was repeated to calcine the sample to temperatures at $100^{\circ}$ intervals to $1100^{\circ} \mathrm{C}$.

\section{Catalytic Characterization}

After physical characterization, we performed preliminary catalytic studies on the alumina aerogels using a custom-built in-house system that is shown schematically in Fig $1{ }^{19}$ The system used a synthetic gas blend (Praxair BAR $97 \mathrm{LOW}$, emission 32 blend: $200 \mathrm{ppm} \mathrm{C}_{3} \mathrm{H}_{8}, 0.5 \% \mathrm{CO}, 6 \% \mathrm{CO}_{2}, 300$ ppm NO, and $\mathrm{N}_{2}$ as the balance) mixed with compressed air at a $0.017: 1$ air to blend ratio ( 1.7\% air) to simulate stoichiometric gasoline automotive engine exhaust. For each test, about $0.3 \mathrm{~g}$ of aerogel material was crushed to a coarse powder and packed into the cylindrical reaction chamber (4.0-cm length by 2.2$\mathrm{cm}$ inner diameter). Inline and tape heaters were used to heat the gas and the temperature in the test section was monitored using thermocouples located 100-mm upstream and 40-mm downstream of the

reactor. An EMS Model 8000 exhaust gas analyzer was used to measure the concentrations of 
hydrocarbons, carbon monoxide, carbon dioxide, nitrogen oxide, and oxygen. Catalytic tests were performed from $300-550^{\circ} \mathrm{C}$ at a space velocity of $20 \mathrm{~s}^{-1}$.

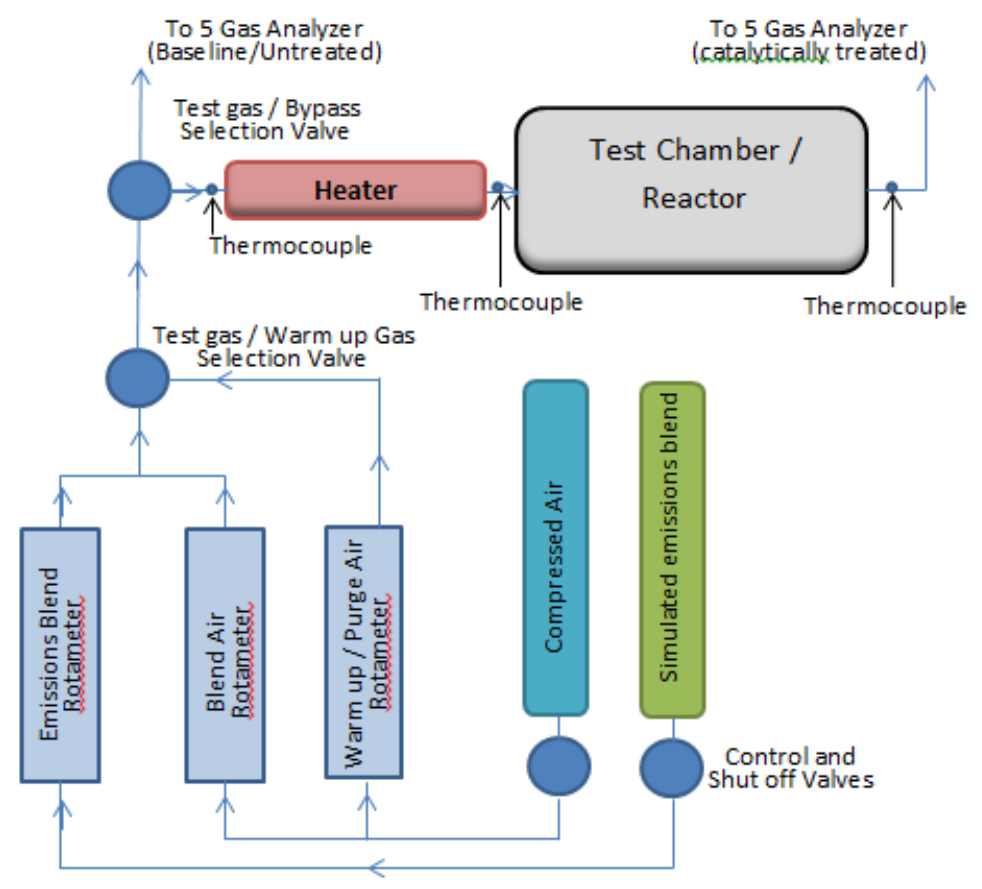

Figure 1. Schematic of simple catalytic testbed.

\section{Uncertainty Estimates}

An uncertainty estimate for each variable was determined based on repeated testing or on instrument specifications. These are summarized in Table 3.

Table 3. Estimates of Measurement Uncertainty.

\begin{tabular}{|c|c|c|c|}
\hline Measurement & $\begin{array}{l}\text { Typical } \\
\text { Value }\end{array}$ & $\begin{array}{l}\text { Uncertainty } \\
\text { Estimate }\end{array}$ & Source of Uncertainty Estimate \\
\hline Bulk Density (g/mL) & $0.04-0.3$ & $.005-.03$ & $\begin{array}{l}\text { calculated from mass \& volume } \\
\text { measurement uncertainty }\end{array}$ \\
\hline Skeletal Density (g/mL) & $1.8-4.4$ & 0.08 & standard deviation \\
\hline BET Surface Area $\left(\mathrm{m}^{2} / \mathrm{g}\right)$ & $240-790$ & $10-50$ & $\begin{array}{l}\text { mass measurement } \& \text { instrument } \\
\text { precision }\end{array}$ \\
\hline Peak Diameter (nm) & $10-20$ & 2 & instrument precision \\
\hline HC Conversion (ppm) & $140-200$ & 4 & instrument accuracy \\
\hline NO Conversion (ppm) & $250-300$ & 25 & instrument accuracy \\
\hline CO Conversion (\%) & $0.4-0.6$ & 0.06 & instrument accuracy \\
\hline
\end{tabular}




\section{RESULTS \& DISCUSSION}

\section{Visual Observations}

Alumina aerogels prepared with two or more solvent exchanges are uniformly transparent in appearance (Figure 2).

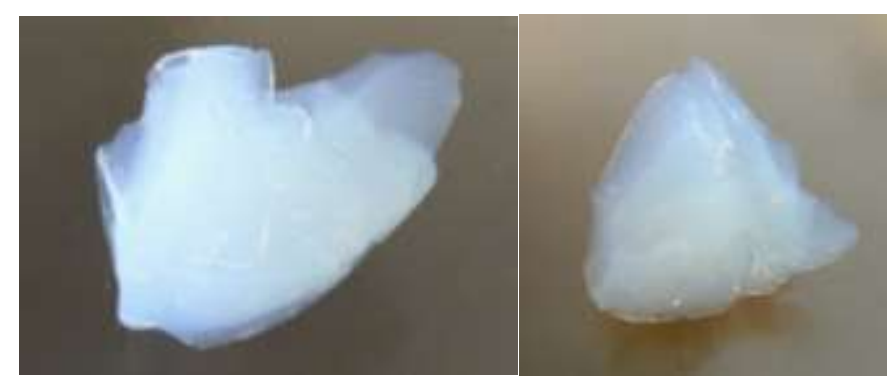

Figure 2. Photographs of alumina RSCE aerogel pieces. The largest dimension of the pieces shown is ca. $2 \mathrm{~cm}$.

\section{Physical and Textural Properties}

The physical and textural properties of the alumina aerogel samples were measured through crude bulk density measurements, the use of helium pycnometry to find skeletal density, and BET surface area and $\mathrm{BJH}$ pore size distribution analysis of $\mathrm{N}_{2}$ sorption isotherms.

Bulk and skeletal density results for the alumina aerogels made with different solvents are shown in Table 4a. The aerogels made with ethanol and methanol have low density and high surface area and compare reasonably well to values in the literature. ${ }^{3}$ The accepted range for the skeletal density of alumina is between 2.5 and $4.0 \mathrm{~g} / \mathrm{mL}$, depending on the particular connectivity of the solid as well as its level of hydration. In the only recipe incorporating water as part of the reaction solvent mixture, the skeletal density is above $3 \mathrm{~g} / \mathrm{mL}$, suggesting a high level of hydration. The recipe using 2-propanol as a solvent has a higher skeletal density still, suggesting that some 2-propanol may remain trapped in the aerogel network. The alumina aerogels prepared with methanol gave the highest surface area; those prepared with 2-propanol the lowest. This suggests that the solvent alcohol has a subtle effect on formation of the sol-gel network. 
Bulk and skeletal density results for several sets of alumina aerogels made with varying numbers of absolute ethanol solvent exchanges are shown in Table 4b.

Table 4a. The effects of solvent type on the physical properties of alumina aerogels made with one solvent exchange.

\begin{tabular}{|l|c|c|c|}
\hline Solvent Employed & Bulk Density $^{\mathrm{a}}$ & $\begin{array}{c}\text { Skeletal } \\
\text { Density }^{\mathrm{b}}\end{array}$ & Surface Area $^{\mathrm{c}}$ \\
\hline Methanol & $0.05 \pm 0.01$ & $2.56 \pm 0.08$ & $790 \pm 50$ \\
\hline Ethanol & $0.07 \pm 0.01$ & $2.75 \pm 0.08$ & $630 \pm 30$ \\
\hline Ethanol \& $\mathrm{DI} \mathrm{H}_{2} \mathrm{O}$ & $0.28 \pm 0.03$ & $3.12 \pm 0.08$ & $250 \pm 10$ \\
\hline 2-Propanol & $0.19 \pm 0.02$ & $4.32 \pm 0.08$ & $240 \pm 10$ \\
\hline
\end{tabular}

Table 4b. Physical and Textural Properties of Alumina aerogels made with additional ethanol exchanges.

\begin{tabular}{|c|c|c|c|}
\hline $\begin{array}{c}\text { Total \# of } \\
\text { Solvent } \\
\text { Exchanges }\end{array}$ & Bulk Density $^{\mathrm{a}}$ & $\begin{array}{c}\text { Skeletal } \\
\text { Density }\end{array}$ & Surface Area $^{\mathrm{c}}$ \\
\hline 3 & $0.044 \pm 0.006$ & $1.86 \pm 0.08$ & $670 \pm 20$ \\
\hline 5 & $0.044 \pm 0.006$ & -- & $700 \pm 30$ \\
\hline 9 & $0.043 \pm 0.005$ & $1.95 \pm 0.07$ & $720 \pm 20$ \\
\hline $3^{*}$ & $0.09 \pm 0.01$ & $2.39 \pm 0.08$ & $620 \pm 20$ \\
\hline
\end{tabular}

Uncertainty value is estimated from ${ }^{\mathrm{a}}$ mass and volume measurement uncertainties propagated over 5 trials; ${ }^{\mathrm{b}}$ mass measurement uncertainty and volume measurement standard deviation after 10+ trials; and " mass measurement uncertainty and instrument precision. * = sample analyzed after catalytic testing.

Solvent exchanges remove excess reagents and byproducts from the epoxide-assisted gelation reaction from the sol-gel matrix. Such impurities, if not removed, might impact the sol-gel matrix itself, as well as the efficacy of the supercritical extraction process. The number of solvent exchanges has no discernable effect on the bulk and skeletal density of the materials. There is a small but statistically significant increase in the surface area with additional solvent exchanges.

Barrett-Joyner-Halenda $(\mathrm{BJH})$ pore size calculations were employed to determine the pore size distributions of aerogel samples. The pore distribution for an alumina aerogel prepared with three ethanol exchanges (Figure 3) shows that the aerogel has pores in the $10-150 \mathrm{~nm}$ range with peak pore volume occurring at a pore diameter of about $37 \mathrm{~nm}$. 


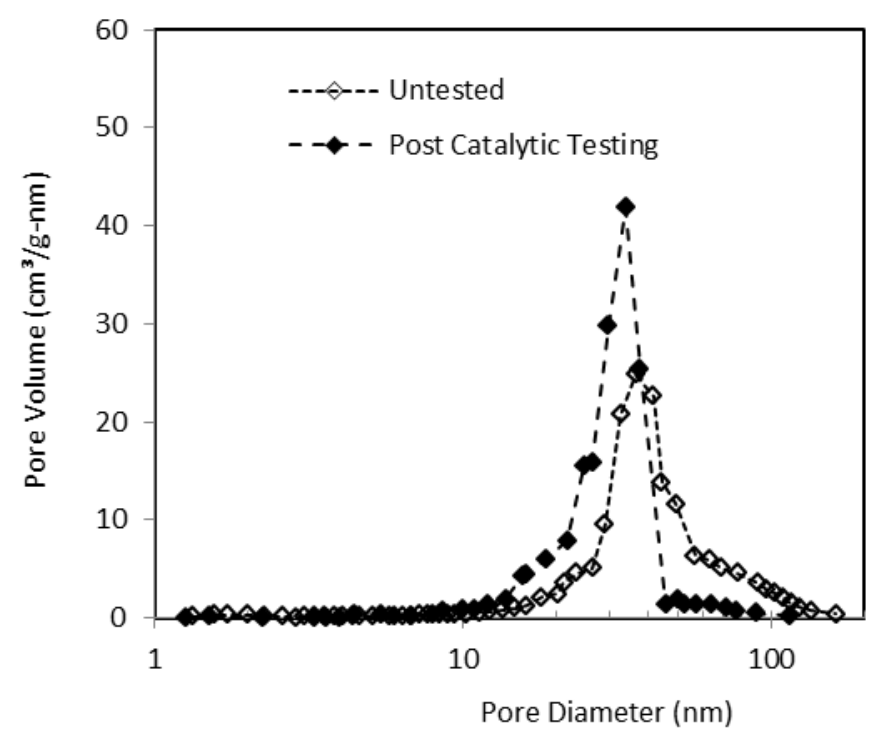

Figure 3. BJH pore size distributions for alumina aerogels (prepared with three ethanol exchanges) obtained from desorption isotherms that employed a 20-s equilibration time: (open symbols) untested aerogel material, (filled symbols) following catalytic testing.

\section{Molecular Composition}

\section{Infrared Spectroscopy}

Fourier-transform infrared (FTIR) spectroscopy was employed to identify the functional groups in the alumina aerogels. In the spectrum of the sample processed following one solvent exchange (see Figure 4a), peaks are observed at 3319 and $3082 \mathrm{~cm}^{-1}$ that are characteristic of asymmetric and symmetric AlO-H stretching in the boehmite structure, respectively. ${ }^{27-29}$ No obvious $\mathrm{C}-\mathrm{H}$ bond stretches are observed. A minor absorption peak at $1164 \mathrm{~cm}^{-1}$, as well as peaks at 1072, 868, and $772 \mathrm{~cm}^{-1}$ can be attributed to the deformation of interlayer AlO-H groups in boehmite. ${ }^{27-29}$ Octahedral Al-O stretching produces a peak at $608 \mathrm{~cm}^{-1} \cdot{ }^{28,30}$ Overlapping peaks observed near $465 \mathrm{~cm}^{-1}$ correspond to skeletal modes of Al-O layers. ${ }^{28}$

NOTE: Figure 4 is included in this submission as a separate PowerPoint file.

Figure 4. FTIR attenuated total reflectance spectra of alumina aerogels prepared with (a) one solvent exchange, (b) three solvent exchanges, (c) three solvent exchanges and subsequent drying to remove adsorbed solvent. Spectra (b) and (c) are offset on the y-axis by $10 \%$ and $25 \%$. respectively, for clarity. 
Peaks due to adsorbed solvent (O-H and C-H stretching modes) are observed in the IR spectra of aerogels processed following additional solvent exchanges (Figure 4b). The two characteristic peaks of boehmite AlO-H interlayer deformation at 3295 and $3092 \mathrm{~cm}^{-1}$ are not observed, and we initially attributed this to overlap with the broad solvent O-H absorption. ${ }^{27-29}$ The three-solvent-exchanged sample was degassed under vacuum at $200^{\circ} \mathrm{C}$ overnight, which facilitated desorption of volatile species. As expected, the FTIR spectrum (Figure 4c) of the dried aerogel does not include the broad $\mathrm{O}-\mathrm{H}$ stretching peak due to adsorbed ethanol at $\sim 3400 \mathrm{~cm}^{-1}$, indicating that that solvent was removed. More surprising is the absence of interlayer $\mathrm{AlO}-\mathrm{H}$ deformation peaks; this implies that the alkyl groups lie in the interlayer space, which would expand the spacing between the layers and thereby inhibit AlO-H interlayer interaction. This type of alkylation of alumina is typical in alkoxyalumoxanes, which are usually formed by solvothermal reaction of aluminum alkoxides in alcoholic media. ${ }^{31}$ Moreover, characteristic $\mathrm{C}-\mathrm{H}$ stretching and bending peaks around 2972 and $1114 \mathrm{~cm}^{-1}$ are present in the spectrum of the degassed aerogel, which suggests that alkyl groups are present within the aerogel matrix. After desorption, there are two additional changes observed in the IR spectrum. First is the emergence of a weak peak at $3645 \mathrm{~cm}^{-1}$ and the second is a shift of the peak at $868 \mathrm{~cm}^{-1}$ to $850 \mathrm{~cm}^{-1}$. Both of these observations are attributed to vibrations of hydroxyl groups at the surface of the alumina, which are freed from hydrogen-bonding upon desorption of the solvent species. ${ }^{27}$

\section{Powder X-Ray Diffraction}

Powder XRD patterns were used to identify crystalline species. The XRD pattern of an alumina aerogel sample prepared with five solvent exchanges is shown in Figure 5 (bottom line). This sample displays Bragg diffraction peaks $(2 \theta, \mathrm{Co}-\mathrm{K} \alpha)$ at 31,59 , and $77^{\circ}$, as well as broad diffraction peaks located approximately at 46 and $85^{\circ}$. These peaks agree well with the characteristic XRD pattern for gelatinous boehmite or pseudoboehmite. ${ }^{3,28,32}$ Pseudoboehmite is a poorly crystallized form of boehmite due to excess water contained in the structure $\left(\mathrm{AlOOH} \cdot 1.7 \mathrm{H}_{2} \mathrm{O}\right) \cdot{ }^{3,28,32,33}$ The peak at $2 \theta=9^{\circ}$ is the characteristic XRD peak of an alkoxyalumoxane prepared using ethanol. ${ }^{31,32}$ Alkoxyalumoxane materials have XRD patterns which are similar to pseudoboehmite, but with larger interlayer spacings due to the presence of 
alkyl groups between the layers of the structure. ${ }^{31,32}$ It is the expansion of the interlayer spacing which gives rise to the characteristic peak below $2 \theta=10^{\circ}$; this peak shifts depending on the length of the alkyl chain of the alcohol used. ${ }^{31}$ These XRD data, coupled with FTIR data, provide evidence that RSCE alumina aerogels prepared with additional solvent exchanges contain alkoxyalumoxane structures.

NOTE: Figure 5 is included in this submission as a separate PowerPoint file.

Figure 5. Overlaid XRD patterns of alumina aerogel prepared with five ethanol exchanges unheated ("as prepared") and after calcining at $100{ }^{\circ} \mathrm{C}$ intervals. Scans are offset on y-axis for clarity.

The phase transformations of this alumina aerogel were investigated by calcining the sample in an oven at $100{ }^{\circ} \mathrm{C}$ intervals and taking XRD patterns. The XRD patterns are overlaid in Figure 5. The characteristic alkoxyalumoxane peak disappears from the XRD pattern after heating to $400^{\circ} \mathrm{C}$. This temperature coincides with a phase transformation to an amorphous alumina form at $400^{\circ} \mathrm{C}$, and appears to be amorphous by $500^{\circ} \mathrm{C}$. By $1100^{\circ} \mathrm{C}$, the XRD pattern most closely resembles that of the theta form of alumina, by comparison with alumina aerogel XRD patterns published by Keysar et al. ${ }^{33}$ This result agrees with data presented by Paglia ${ }^{34}$ and Wefers et al., ${ }^{35}$ that suggest that gelatinous boehmite can transition to theta-alumina between $800^{\circ} \mathrm{C}^{34}$ and $1100^{\circ} \mathrm{C}^{35}$

\section{Morphology}

Scanning Electron Microscopy

Scanning electron microscopy (SEM) was employed to investigate the morphology of the alumina aerogel samples. Images of alumina aerogel samples made with one and three solvent exchanges are shown in Figure 6. The images show an interconnected, fibrous microstructure similar to that reported by Baumann et al. ${ }^{3}$ for alumina aerogels prepared using an epoxide-assisted mechanism from an ethanolic $\mathrm{AlCl}_{3} \cdot 6 \mathrm{H}_{2} \mathrm{O}$ with $\mathrm{CO} 2$ supercritical drying. ${ }^{3}$ This is also similar to the microstructure typically reported for alumina aerogels prepared from alkoxide precursors. ${ }^{3,4,13,33}$ 


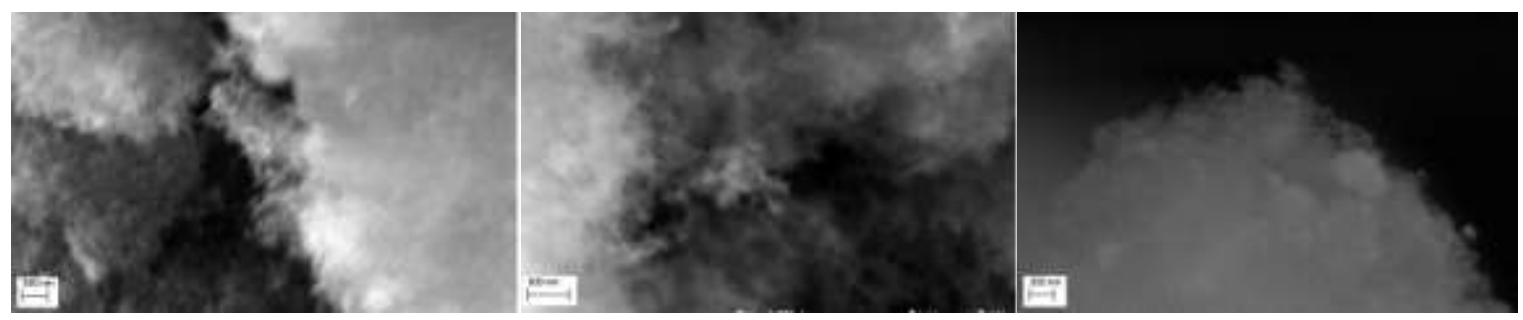

Figure 6. SEM images of alumina aerogels prepared using (a) one solvent exchange, (b,c) three solvent exchanges. Aerogel in view (c) has undergone testing in catalytic test bed (heating to $575^{\circ} \mathrm{C}$ ). Imaging parameters for (a) magnification 49kX, EHT $15 \mathrm{kV}$, working distance $10 \mathrm{~mm}$; (b) magnification 84kX, EHT $5 \mathrm{kV}$, working distance $8.5 \mathrm{~mm}$; (c) $49 \mathrm{kX}$, EHT $15 \mathrm{kV}$, working distance $6.5 \mathrm{~mm}$.

Energy-dispersive x-ray spectroscopy was employed in order to verify good elemental dispersion in aerogel samples. As was expected for an alumina structure, there was an even distribution of oxygen and aluminum atoms throughout the sample. Small signals due to carbon and chlorine atoms were also observed.

\section{GC-MS Headspace Analysis of Solvent}

GC-MS analysis was performed on solvent samples from each of nine sequential solvent exchanges. Headspace sampling was utilized to analyze volatile components extracted from the gel without the risk of clogging the GC column with bits of gel or nonvolatile components. The results of GC-MS headspace analysis of the first post-gelation solvent exchange sample are shown in Figure 7. Each GC peak was attributed to a specific chemical species by analyzing mass spectra with the help of a computer database. Peaks due to unretained gas-phase species (air), methanol (used to clean the autosampler syringe), ethanol (the solvent) and propylene oxide (excess reagent) are clearly resolved. Two of the peaks from $t=2$ to 4 min appear to be due to side products. 1-chloropropan-2-ol and 2chloropropan-1-ol (Scheme 2) are products of an irreversible ring-opening reaction after a propylene oxide molecule has participated in the hydrolysis of an aluminum ionic complex. ${ }^{23}$ Assuming that the peak intensities for these isomers are qualitatively representative of their concentrations in the solvent solution, these data show that 1-chloropropan-2-ol is the primary product of the irreversible ring-opening 
reaction (Scheme 2). Peaks attributed to ethyl acetate and methyl isobutyl ketone, employed as ethanol denaturants, were also detected.

NOTE: Figure 7 is included in this submission as a separate PowerPoint file.

Figure 7. GC-MS headspace analysis of solvent sample following the first post-gelation exchange, showing total ion abundance versus retention time ( $\mathrm{min})$.

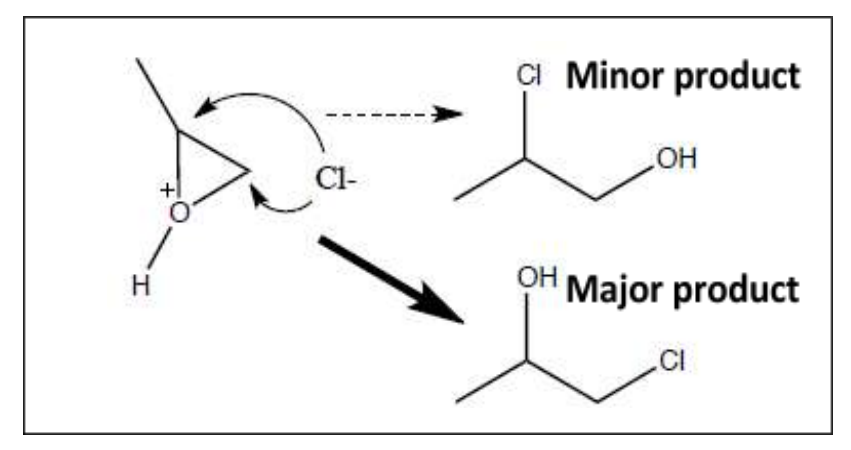

Scheme 2. Major and minor products of the irreversible epoxide ring-opening side reaction, as determined by GCMS headspace analysis.

Performing additional solvent exchanges with absolute ethanol serves to remove propylene oxide and reaction byproducts from the sol-gel sample. For purposes of comparison, the percentage of the total GC-MS headspace analysis peak area in each of nine sequential exchanges that is due to each species was calculated and plotted (Figure 8). The relative amount of propylene oxide signal decreases with each solvent exchange; no discernible peak is observed after five solvent exchanges. Five solvent exchanges are required to effectively eliminate excess reagents and byproducts from the sample. 

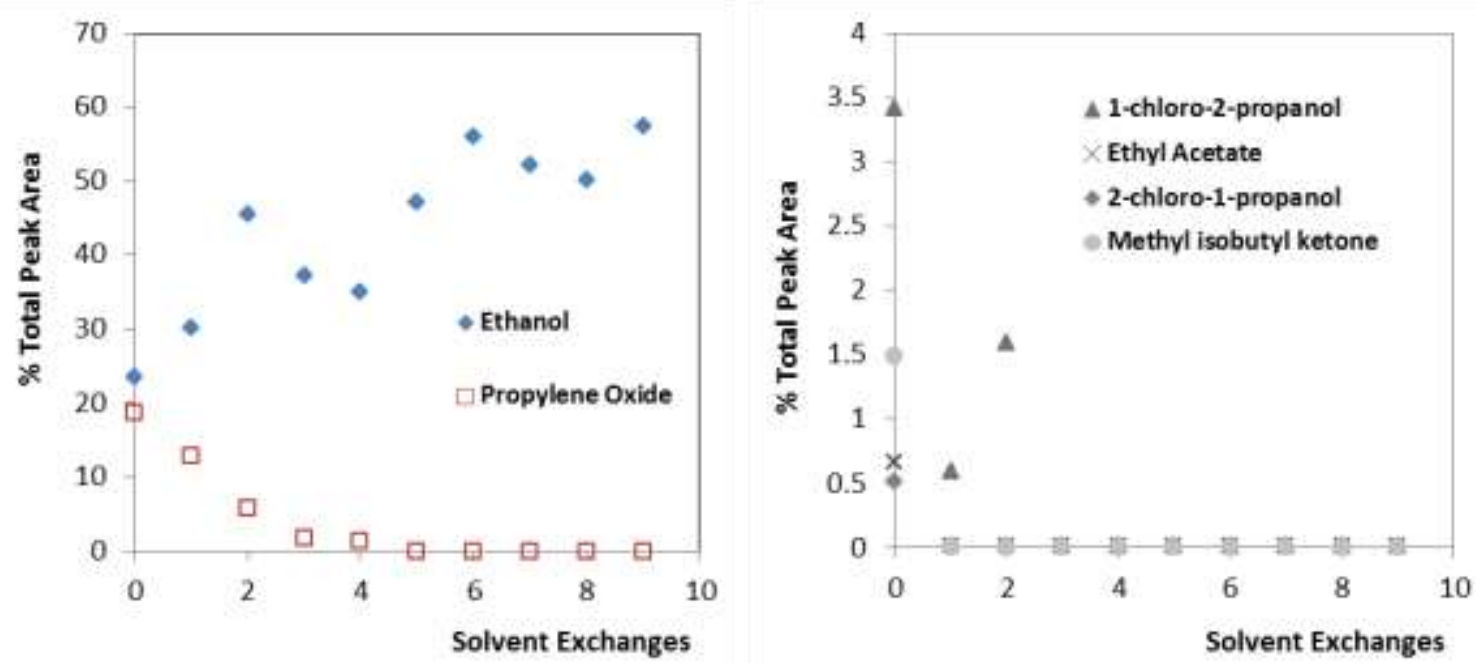

Figure 8. Percent of total peak area for chemical species detected by GC-MS headspace analysis of solvent exchanges. Estimated relative uncertainty for each point is $<10 \%$.

\section{Catalytic Characterization of Alumina-Based Aerogels}

Catalytic characterization of the alumina aerogels was conducted using a constant space velocity of $20 \mathrm{~s}^{-1}$ over the temperature range $200-550^{\circ} \mathrm{C}$. Figure 9 shows two trials of constant space velocity catalytic testing using fresh aerogel as a catalyst in each trial. 

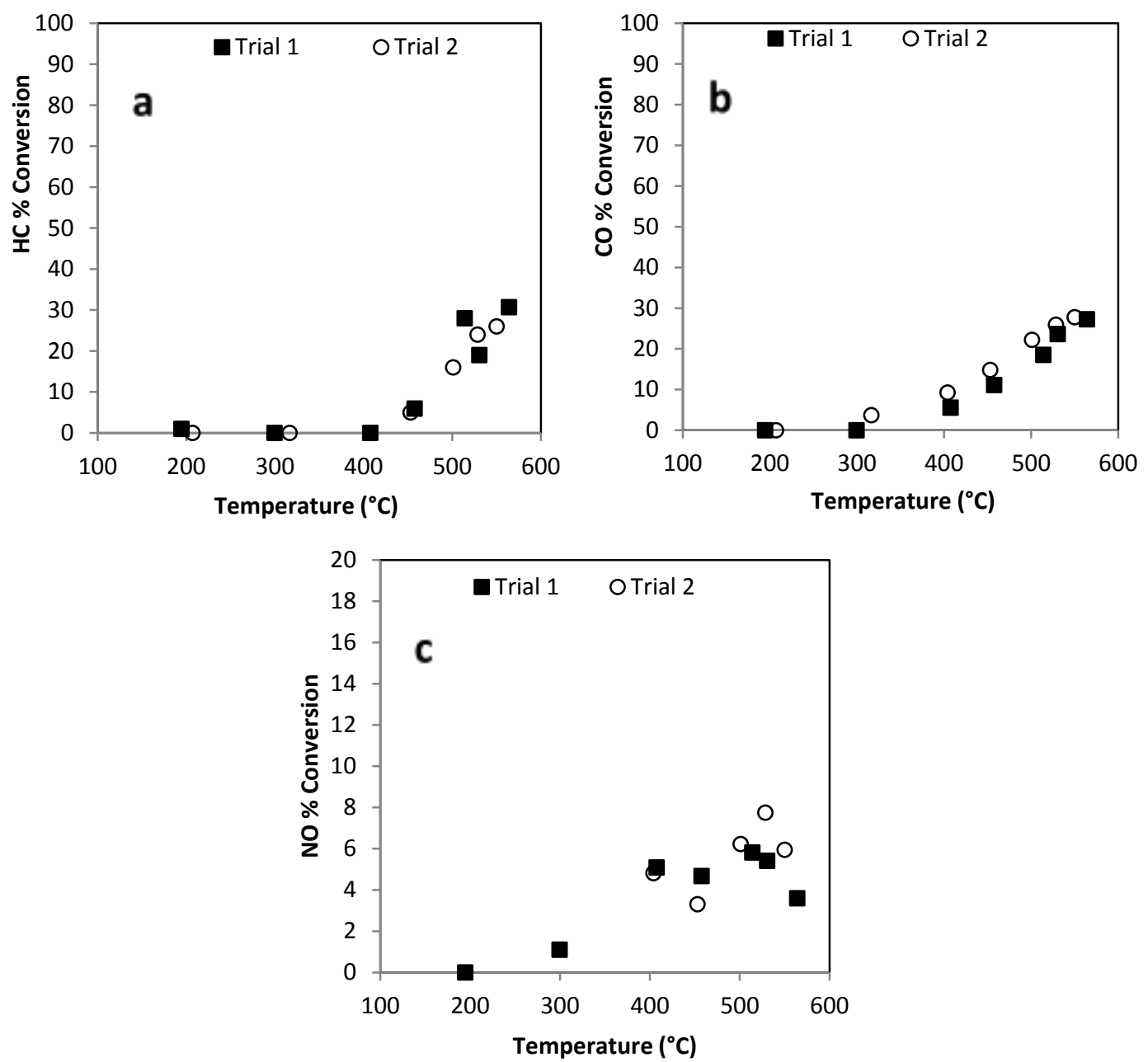

Figure 9. Percent conversion of (a) $\mathrm{HC}$, (b) $\mathrm{CO}$, and (c) $\mathrm{NO}$ when employing fresh alumina aerogel as a catalyst under constant space velocity conditions over two trials. This figure shows results of two replicate runs in order to provide a visual indication of the run-to-run uncertainty. Uncertainty estimates for measurements made with the five-gas analyzer are given in Table 3.

The alumina aerogel catalyst starts to show catalytic activity at $400{ }^{\circ} \mathrm{C}$, and reaches maximum conversions of $31 \% \mathrm{HC}, 28 \% \mathrm{CO}$, and $6 \% \mathrm{NO}$ (Figure 9). These results show that alumina aerogels have some catalytic activity. When operated at stoichiometric conditions and similar space velocities to the ones examined here, a conventional catalytic converter (i.e., one containing catalysts based on some combination of platinum, palladium and rhodium) would provide conversion efficiencies of $90 \%$ or more. ${ }^{36,37}$ Conventional precious metal catalysts also show "light off temperatures" (defined as the temperature at which they first achieve $50 \%$ conversion) at values in the $250{ }^{\circ} \mathrm{C}$ range. ${ }^{37,38}$ The activity of 
the unoptimized aerogel catalysts described here should be considered in light of the significant potential cost and environmental benefits of eliminating the need for platinum group metals.

Alumina-based aerogels were physically characterized before and after catalytic testing. These values are included in Table $4 \mathrm{~b}$. It should be noted that crude bulk densities measured after catalytic testing may be artificially high because samples were not degassed; therefore adsorbed species from the simulated automotive gas blend might contribute to the measured mass. After catalytic testing (heating to a temperature of $575{ }^{\circ} \mathrm{C}$ ) there is a decrease in the number of pores with diameters greater than $40 \mathrm{~nm}$ and an increase in the number of pores with diameters below $40 \mathrm{~nm}$ (see Figure 3). This is likely due to pore collapse caused by sintering and/or effects of exposure to exhaust gases under high temperatures.

SEM imaging was employed to examine the morphology of alumina-based aerogel samples before and after catalytic testing (see Figure 6). The dense, fibrous nanomorphology of the alumina aerogel remains after catalytic testing to $550^{\circ} \mathrm{C}$, indicating that the alumina does not change significantly under these conditions.

\section{CONCLUSIONS}

High-quality alumina-based aerogels can be readily prepared via an epoxide-assisted gelation reaction followed by rapid supercritical extraction (RSCE).

FTIR and XRD analyses show that alumina-based RSCE aerogels are generally amorphous and contain boehmite derivatives. Aerogel samples prepared with a single solvent exchange prior to processing have a pseudoboehmite structure, whereas those prepared with additional solvent exchanges show evidence of an alkoxyalumoxane structure. BJH pore size distribution analysis reveals a primarily mesoporous structure in all alumina aerogels. SEM imaging shows that alumina-based RSCE aerogels exhibit characteristic morphologies that agree with literature reports of alumina aerogels prepared by lower-temperature $\mathrm{CO}_{2}$ extraction in a critical point dryer. In addition, an increase in textural porosity was observed due to additional solvent exchanges, which may have a positive effect on the catalytic activity of 
the samples. EDX investigations of alumina-based aerogels reveal uniform elemental distribution across the samples at the micro-scale.

Solvent characterization by GC-MS headspace analysis shows that excess propylene oxide, chloropropanol products of an irreversible epoxide ring-opening reaction, and denaturants are present in the alumina gel following gelation. Although solvent exchanges are not required for the RSCE method, we found that performing five solvent exchanges, which removes these compounds, results in increased aerogel surface area. This suggests that the presence of compounds other than ethanol in the pores of the wet gel has a direct impact on the textural properties of the resulting aerogel, most likely due to the impact of the supercritical point of the mixture present in the pores of the aerogel during RSCE. Unless the solvent in the sol-gel pores becomes a supercritical fluid, the extraction process will release some solvent as a vapor, resulting in the collapse of the smaller pores, which experience greater surface tension and capillary effects. Textural porosity may prove to be important in improving catalytic activity. ${ }^{39}$

Preliminary catalytic temperature sweep tests demonstrate that alumina RSCE aerogels show moderate ability to catalyze reactions to remove unburned hydrocarbons, carbon monoxide, and nitrogen oxide from automotive exhaust. Ongoing work in our laboratory is focused investigation of mixed aerogel materials (including nickel-alumina and cobalt-alumina aerogels) for catalytic applications. ${ }^{18,19}$

\section{ACKNOWLEDGEMENTS}

This material is based upon work supported by the National Science Foundation (NSF) under Grants No. CHE-0847901 and DMR-1206631. The authors thank Michael E. Hagerman for helpful discussions, Justin E. Rodriguez for assistance with the catalytic test bed measurements, and the Union College Geology Department for use of the XRD instrument. SJJ and NJHD are grateful for summer funding from the Union College Summer Research Fellowship Program. Additional support of this project was provided through the Union College Faculty Research Fund, Student Research Grant and Presidential Green Grant programs. The SEM instrument was funded through grants from the National Science Foundation (NSF MRI 0619578) and New York State Assembly RESTORE-NY. 


\section{REFERENCES}

1. W. Amatayakul and O. Ramnäs, J. Clean Prod, 2001, 9(5), 395-403.

2. G. Pajonk, Catalysis Today, 1997, 35, 319-337.

3. T. F. Baumann, A. E. Gash, S. C. Chinn, Sawvel. A.M., R. S. Maxwell and J. H. J. Satcher, Chem. Materials, 2005, 17, 395-401.

4. M. S. Bono, A. M. Anderson and M. K. Carroll, J. Sol Gel Sci. Technol., 2010, 53, 216-226.

5. R. Tleimat-Manzalji, D. Bianchi and G. M. Pajonk, Reaction, 1993, 51, 29-37.

6. H. Hirashima, C. Kojima and H. Imai, J. Sol Gel Sci. Technol., 1997, 8, 843-846.

7. S. Rouanet, Investigation and Characterization of Silica-Alumina Aerogels, Springer, 1995.

8. L. Owens, J. Non Cryst. Solids, 1995, 186, 177-183.

9. R. J. Willey, S. J. Teichner and G. M. Pajonk, J. of Molecular Catalysis, 1992, 77, 201-219.

10. M. Cauqui, J. Calvino, G. Cifredo, L. Esquivias and J. Rodriguezizquierdo, J. Non Cryst. Solids, 1992, 147-148, 758-763.

11. S. Krompiec, J. Non Cryst. Solids, 2003, 315, 297-303.

12. Z. Hao, Q. Zhu, Z. Jiang, B. Hou and H. Li, Fuel Processing Technology, 2009, 90, 113-121.

13. C. Hoang-Van, B. Pommier, R. Harivololona, P. Pichat, J. Non Cryst. Solids, 145, 250-254.

14. Y. Mizushima and M. Hori, J. Mater. Sci., 1995, 30, 1551-1555.

15. B.M. Gauthier, S.D. Bakrania, A.M. Anderson and M.K. Carroll, J. Non Cryst. Solids, 2004, 350, 238-243.

16. M.K. Carroll, A.M. Anderson and C.A. Gorka, JoVE (J. Visualized Experiments), 2014, e51421e51421.

17. L.B. Brown, A.M. Anderson and M.K. Carroll, J. Sol Gel Sci. Technol., 2012, 62, 404-413.

18. N.J.H. Dunn, M.K. Carroll and A.M. Anderson, Polymer Preprints, 2011, 52, 250.

19. B.A. Bruno, J.E. Madero, S.J. Juhl, J.E. Rodriguez, N.J.H. Dunn, M.K. Carroll, and A.M. Anderson, Proceedings of the $9^{\text {th }}$ International Congress on Catalysis and Automotive Pollution Control (CAPoC9), 2012.

20. A.M. Anderson and M. K. Carroll, Aerogels Handbook, 2011, 47.

21. A.M. Anderson, M. K. Carroll, E. C. Green, J. T. Melville and M. S. Bono, J. Sol Gel Sci. Technol., 2010, 53, 199-207.

22. A. M. Anderson, C. W. Wattley and M. K. Carroll, J. Non Cryst. Solids, 2009, 355, 101-108.

23. A. E. Gash, T. M. Tillotson, J. H. Satcher Jr., J. F. Poco, L. W. Hrubesh and R. L. Simpson, Chemistry of Materials, 2001, 13, 999-1007. 
24. L.M. Hair, P.R. Coronado, L.G. Reynolds, J.G., J. Non Cryst. Solids, 2000, 270, 115-122.

25. G. Li, L. Hu and J. M. Hill, Applied Catalysis A: General, 2006, 301, 16-24.

26. G. Reichenauer and G. W. Scherer, J. Non Cryst. Solids, 2001, 285, 167-174.

27. S.A. Dickie, A.J. McQuillan, Langmuir, 2004, 20, 11630-11636.

28. Y. Zhu, Z. Jiang, L. Zhang, J. Shi and D. Yang, Industrial and Engineering Chemistry Research, 2012, 51, 255-261.

29. J.J. Fripiat, H. Bosmans, P.G. Rouxhet, J. Phys. Chem. 1967, 71, 1097-1111.

30. P. Tarte, Spectrochimica Acta. Part A: Molecular Spectroscopy, 1967, 23A, 2127-2143.

31. M. Inoue, M. Kimura and T. Inui, Chem. Materials, 2000, 12, 55-61.

32. A. Corrias, M. F. Casula, A. Falqui and G. Paschina, Chem. Materials, 2004, 16, 3130-3138.

33. S. Keysar, G.E. Shter, Y. de Hazan, Y. Cohen and G.S. Grader, Chem. Materials, 1997, 9, 24642467.

34. G. Paglia, Determination of the structure of $\gamma$-alumina using empirical and first principles calculations combined with supporting experiments. Faculty of Science. Department of Applied Physics and Department of Applied Chemistry. Curtin University of Technology, 2004.

35. K. Wefers and C. Misra, "Oxides and Hydroxides of Aluminum.” Alcoa Laboratories Technical Paper No. 19, Revised ed., Alcoa Laboratories: 1987.

36. R.M. Heck, R.J. Farrauto, and S.T. Gulati, “Catalytic Air Pollution Control Commercial Technology, $3^{\text {rd }}$ ed." Wiley 2009

37. J.B. Heywood, "Internal Combustion Engine Fundamentals" McGraw Hill 1988

38. R. Stone, "Introduction to Internal Combustion Engines, $3^{\text {rd }}$ ed." SAE International 1999

39. Kim, P.; Joo, J.B.; Kim, H.; Kim, W.; Kim, Y.; Song, I.K.; Yi, J. Catal. Lett. 2005, 104, 181-189. 